\title{
Rough Sets in Knowledge Discovery and Data Mining
}

\section{Ning ZHONG*}

\begin{abstract}
Rough set theory constitutes a sound basis for KDD (Knowledge Discovery and Data Mining) to deal with real world problems systematically. In the paper, we investigate several rough sets based hybrid systems that can be used in a multi-phase KDD process to discover patterns hidden in data in many aspects. We also outline the latest researches and give future directions.
\end{abstract}

Keywords: rough sets, data mining, knowledge discovery, hybrid systems, soft computing

\section{Introduction}

Massive data sets in real world have driven research, applications, and tool development in business, science, government, and academia. The continued growth in data collection in all of these areas ensures that the fundamental problem which KDD (knowledge discovery and data mining) ad dresses, namely how does one understand and use one's data, will continue to be of critical importance across a large swath of organizations [9, 47].

There are two major methodologies to deal with the complexity of real world and imperfect data. The first is hybridization. Here "hybridization" means the way of combining many advantages of existing methods, and avoiding their disadvantages or weaknesses when the existing methods are used separately. There are ongoing efforts to integrate logic (including non-classical logic), artificial neural networks, probabilistic and statistical reasoning, fuzzy set theory, rough set theory, genetic algorithm and other methodologies in the soft computing paradigm $[2,24,44]$.

The second methodology is the multi-phase process. The KDD process is usually a multi-phase process involving numerous steps like data preparation, preprocessing, search for hypothesis genera-

* Department of Information Engineering, Maebashi Institute of Technology tion, pattern formation, knowledge evaluation, representation, refinement, and management. Furthermore, the process may be repeated at different stages when database is updated $[9,38,43,46,47]$. The multi-phase process is an important methodology for knowledge discovery from real-life data [43]. Although the process-centric view has recently been widely accepted by researchers in the KDD community, few KDD systems provide the capabilities that a more complete process should possess.

Rough set theory constitutes a sound basis for KDD to deal with complex real-life data. It offers useful tools to discover patterns hidden in data in many aspects $[14,27,31,46]$. It can be used in different phases of a knowledge discovery process like attribute selection, attribute extraction, data reduction, decision rule generation, and pattern extraction (templates, association rules) [12]. Furthermore, recent extensions of rough set theory (rough mereology) have brought new methods for decomposition of large data sets, data mining in distributed and multi-agent based environment, mining in ordered information tables, and granular computing $[28,29,30,34,40]$.

In the paper, we investigate several rough sets based hybrid systems that can be used in a multiphase KDD process to discover patterns hidden in data in many aspects. In a sense, such rule discovery process described in this paper can be regarded as a demonstration of the process-centered KDD metho- 
dology and applications of rough set theory in this process. Section 2 describes a soft hybrid induction system GDT-RS constituting the core in discovery of classification rules from databases with uncertain and incomplete data. The system is based on a combination of Generalization Distribution Table (GDT) and the Rough Set methodology. Furthermore, in Sections 3 and 4 we introduce two systems, Rough Sets with Heuristics (RSH) for attribute selection and Rough Sets with Boolean Reasoning (RSBR) for discretization of real valued attributes, respectively. They are responsible for two steps in the preprocessing realized before the GDT-RS starts. $\mathrm{n}$ in Section 5, we describe how to combine GDT-RS with Inductive Logic Programming (ILP) to extend attribute-value learning into relation learning. Finally, Section 6 gives concluding remarks and future research directions.

\section{Generalization Distribution Table and Rough Sets (GDT -RS)}

GDT-RS is a soft hybrid induction system for discovering classification rules from databases with uncertain and incomplete data $[6,39,44]$. The system is based on a hybridization of Generalization Distribution Table (GDT) and the Rough Set methodology.

The GDT-RS system can generate, from noisy and incomplete training data, a set of rules with the minimal (semi-minimal) description length, having large strength, and covering of all instances.

\subsection{Generalization Distribution Table (GDT)}

We distinguish two kinds of attributes, namely: condition attributes and decision attributes (sometimes called class attributes) in a database. Condition attributes are used to describe possible instances in GDT while the decision attributes correspond to concepts (classes) described in a rule. Usually a single decision attribute is all what is required.

For simplicity, the wild card will be sometimes omitted in the paper

2 How to use background knowledge in the rule discovery process is not discussed since the page limitation. For such discussion, see our papers [4]
Any GDT consists of three components: possible instances, possible generalizations of instances, and probabilistic relationships between possible instances and possible generalizations.

Possible instances, represented at the top row of GDT, are defined by all possible combinations of attribute values from a database. Possible generalizations of instances, represented by the left column of a GDT, are all possible cases of generalization for all possible instances. A wild card “*” denotes the generalization for instances ${ }^{1}$. For example, the generalization $* b_{0} c_{0}$ means that the attribute $a$ is superfluous (irrelevant) for the concept description. In other words, if an attribute $a$ takes values from $\left\{a_{0}, a_{1}\right\}$ and both $a_{0} b_{0} c_{0}$ and $a_{1} b_{0} c_{0}$ describe the same concept, the attribute $a$ is superfluous, i.e., the concept can be described by $b_{0} c_{0}$. Therefore, we use the generalization $* b_{0} c_{0}$ to describe the set $\left\{a_{0} b_{0} c_{0}, a_{1} b_{0}\right.$ $\left.c_{0}\right\}$.

The probabilistic relationships between possible instances and possible generalizations, represented by entries $G_{i j}$ of a given GDT, are defined by means of a probabilistic distribution describing the strength of the relationship between any possible instance and any possible generalization. The prior distribution is assumed to be uniform, if background knowledge is not available ${ }^{2}$. Thus, it is defined by Eq. (1)

$$
\begin{aligned}
G_{i j} & =p\left(P I_{j} \mid P G_{i}\right)= \\
& =\left\{\begin{array}{c}
\frac{1}{N_{P G i}} \text { if } P I_{j} \in P G_{i} \\
0
\end{array}\right.
\end{aligned}
$$

where $P I_{j}$ is the $j$-th possible instance, $P G_{i}$ is the $i$ -th possible generalization, and $N_{P G_{i}}$ is the number of the possible instances satisfying the $i$-th possible generalization, i.e.,

$$
N_{P G_{i}}=\prod_{k \in\left\{l \mid P G_{i},[l \mid * *\}\right.} n_{k}
$$

where $P G_{i}[l]$ is the value of the $l$-th attribute in the possible generalization $P G_{i}$ and $n_{k}$ is the number of values of $k^{\text {th }}$ attribute. Certainly we have $\sum_{j} G_{i j}=1$ for any $i$.

Assuming $E=\prod_{k=1}^{m} n_{k}$ the equation Eq. (1) can be rewritten in the following form: 


$$
\begin{aligned}
G_{i j} & =p\left(P I_{j} \mid P G_{i}\right) \\
& =\left\{\begin{array}{cl}
\frac{k \in\left\{l \mid P G_{i}[l] \neq *\right\}}{E} & \text { if } P I_{j} \in P G_{i} \\
0 & \text { otherwise }
\end{array}\right.
\end{aligned}
$$

Furthermore, rule discovery can be constrained by three types of biases corresponding to three components of the GDT so that a user can select more general concept descriptions from an upper level or more specific ones from a lower level, adjust the strength of the relationship between instances and their generalizations, and define/ select possible instances [39].

\subsection{Rule Strength}

Let us recall some basic notions for rule discovery from databases represented by decision tables [12]. A decision table (DT) is a tuple $T=(U, A, C, D)$, where $U$ is a nonempty finite set of objects called the universe, $A$ is a nonempty finite set of primitive attributes, and $C, D \subseteq A$ are two subsets of attributes that are called condition and decision attributes, respectively $[27,31]$. By $I N D(B)$ we denote the indiscernibility relation defined by $B \subseteq A,[x]_{I N D}$ B) denotes the indiscernibility (equivalence) class defined by $x$, and $U / B$ the set of all indiscernibility classes of $I N D(B)$. A descriptor over $B \subseteq A$ is any pair $(a, v)$ where $a \in A$ and $v$ is a value of $a$. If $P$ is a conjunction of some descriptors over $B \subseteq A$ then by $[P]_{B}$ (or $[P]$ ) we denote the set of all objects in $D T$ satisfying $P$.

In our approach, the rules are expressed in the following form:

$$
P \rightarrow Q \text { with } S
$$

i.e., "if $P$ then $Q$ with the strength $S$ " where $P$ denotes a conjunction of descriptors over $C$ (with non-empty set $[P]_{D T}$ ), $Q$ denotes a concept that the rule describes, and $S$ is a "measure of strength" of the rule defined by

$$
S(P \rightarrow Q)=s(P) \times(1-r(P \rightarrow Q))
$$

where $s(P)$ is the strength of the generalization $P$ (i.e., the condition of the rule) and $r$ is the noise rate function. The strength of a given rule reflects the incompleteness and uncertainty in the process of rule inducing influenced both by unseen instances and noise.

The strength of the generalization $P=P G$ is given by Eq. (5) under that assumption that the prior distribution is uniform

$$
s(P)=\sum_{l} p\left(P I_{l} \mid P\right)=\operatorname{card}\left([P]_{D T}\right) \times \frac{1}{N_{P}}
$$

where $\operatorname{card}\left([P]_{D T}\right)$ is the number of observed instances satisfying the generalization $P$.

The strength of the generalization $P$ represents explicitly the prediction for unseen instances. On the other hand, the noise rate is given by Eq. (6)

$$
r(P \rightarrow Q)=1-\frac{\operatorname{card}\left([P]_{D T} \cap[Q]_{D T}\right)}{\operatorname{card}\left([P]_{D T}\right)}
$$

It shows the quality of classification measured by the number of instances satisfying the generalization $P$ which cannot be classified into class $Q$. The user can specify an allowed noise level as a threshold value. Thus, the rule candidates with the larger noise level than a given threshold value will be deleted.

One can observe that the rule strength we are proposing is equal to its confidence [1] modified by the strength of the generalization appearing on the left hand side of the rule. The reader can find in literature other criteria for rule strength estimation (see e.g., $[3,17,11]$ ).

\subsection{Simplification of the Decision Table by GDT- RS}

The process of rule discovery consists of decision table preprocessing including of selection and extraction of relevant attributes (features) and the relevant decision rule generation. The relevant decision rules can be induced from the minimal rules (i.e., with the minimal length of their left hand sides with respect to the discernibility between decisions) by tuning them (e.g., drooping some conditions to obtain more general rules better predisposed to classify new objects even if they not classify properly some objects from the training set). The relevant rules can be induced from the set of all minimal rules or its subset covering the set of objects of a given decision table $[12,31]$. A representative 
approach for the problem of generation of the so called local relative reducts of condition attributes is the one to represent knowledge to be preserved about the discernibility between objects by means of the discernibility functions [27,31].

It is obvious that by using the GDT one instance can be matched by several possible generalizations, and several instances can be generalized into one possible generalization. Simplifying a decision table by means of the GDT-RS system leads to a minimal (or sub-minimal) set of generalizations covering all instances. The main goal is to find a relevant (i.e., minimal or semi-minimal with respect to the description size) covering of instances still allowing to resolve conflicts between different decision rules recognizing new objects. The first step in the GDT - RS system for decision rules generation is based on computing of local relative reducts of condition attributes, by means of discernibility matrix method $[4,27,31]$. Moreover, instead of searching for dispensable attributes we are rather searching for relevant attributes using a bottom-up method.

Any generalization matching instances with different decisions should be checked by means of Eq. (6). If the noise level is smaller than a threshold value, such generalization is regarded as a reasonable one. Otherwise, the generalization is contradictory.

Furthermore, a rule, in GDT-RS, is selected according to its priority. The priority can be defined by the number of instances covered (matched) by a rule (i.e., the more instances are covered, the higher the priority is), by the number of attributes occurring on the left hand side of rule (i.e., the less the attribute number is, the higher the priority is), or by the rule strength [39].

We have developed two algorithms (called "Optimal Set of Rules" and "Sub-Optimal Solution") for the GDT-RS implementation. The algorithms are not discussed since the page limitation. For such discussion, see our papers [6, 44].

\section{Rough Sets with Heuristics (RSH)}

$\mathrm{RSH}$ is a system for attribute subset selection. It is based on rough sets with heuristics [45]. The development of the RSH is based on the following observations: (i) a database always contains a lot of attributes that are redundant and not necessary for rule discovery; (ii) if these redundant attributes are not removed, not only the time complexity of rule discovery increases, but also the quality of the discovered rules may be significantly decreased.

The goal of attribute selection is to find an optimal subset of attributes according to some criterion, so that a classifier with the highest possible accuracy can be induced by an inductive learning algorithm using information about data available only from the subset of attributes.

\subsection{Rough Sets with Heuristics}

In this section we explain some concepts of rough sets related to attribute selection in preprocessing [27].

Let $C$ and $D$ denote the condition and decision attribute sets of the decision table $T$, respectively. The $C$-positive region of $D$ is the set of all objects from the universe $U$ which can be classified with certainty to classes of $U / D$ employing attributes from $C$, i.e.,

$$
\operatorname{POS}_{\mathrm{C}}(D)=\bigcup_{X \in U / D} C X
$$

where $\underline{C X}$ denotes the lower approximation of the set $X$ with respect to $C$, i.e., the set of all objects from $U$ that can be with certainty classified as elements of $X$ basing on attributes from $C$.

An attribute $c(c \in C)$ is dispensable in a decision table $T$, if $P O S_{(C-\{c\})}(D)=P O S_{\mathrm{C}}(D)$; otherwise attribute $c$ is indispensable in $T$. A set of attributes $R \subseteq C$ is called a reduct of $C$ if it is a minimal attribute subset preserving the condition: $P O S_{R}$ $(D)=P O S_{C}(D)$. Furthermore, the set of all the attributes indispensable in $C$, is denoted by CORE (C). We have

$$
\operatorname{CORE}(C)=\cap R E D(C)
$$

where $R E D(C)$ is the set of all reducts of $C$.

The quality of an attribute subset $R$ in GDT-RS depends on the strength of rules discovered by using this subset. The higher the strength is, the better the subset is. Searching for attributes that are of benefit to acquire the rules with large cover rate and strength is based on the selection strategy described 
Table 1: A sample database

\begin{tabular}{|c|cccc|c|}
\hline$U \backslash A$ & $\mathrm{a}$ & $\mathrm{b}$ & $\mathrm{c}$ & $\mathrm{d}$ & $\mathrm{e}$ \\
\hline $\mathrm{u} 1$ & $a_{1}$ & $b_{0}$ & $c_{2}$ & $d_{1}$ & $e_{1}$ \\
$\mathrm{u} 2$ & $a_{1}$ & $b_{0}$ & $c_{2}$ & $d_{0}$ & $e_{1}$ \\
u3 & $a_{1}$ & $b_{2}$ & $c_{0}$ & $d_{0}$ & $e_{2}$ \\
u4 & $a_{1}$ & $b_{2}$ & $c_{2}$ & $d_{2}$ & $e_{0}$ \\
u5 & $a_{2}$ & $b_{1}$ & $c_{0}$ & $d_{0}$ & $e_{2}$ \\
u6 & $a_{2}$ & $b_{1}$ & $c_{1}$ & $d_{0}$ & $e_{2}$ \\
u7 & $a_{2}$ & $b_{1}$ & $c_{2}$ & $d_{1}$ & $e_{1}$ \\
\hline
\end{tabular}

Table 2: The initial state for attribute selection

\begin{tabular}{|c||c||c|}
\hline$U \backslash A$ & $\mathrm{~b}$ & $\mathrm{e}$ \\
\hline $\mathrm{u} 3$ & $b_{2}$ & $e_{2}$ \\
$\mathrm{u} 4$ & $b_{2}$ & $e_{0}$ \\
$\mathrm{u} 5$ & $b_{1}$ & $e_{2}$ \\
$\mathrm{u} 6$ & $b_{1}$ & $e_{2}$ \\
$\mathrm{u} 7$ & $b_{1}$ & $e_{1}$ \\
\hline
\end{tabular}

Table 3: Selecting the second attribute from $\mathrm{R}=\{\mathrm{a}, \mathrm{c}, \mathrm{d}\}$

\begin{tabular}{|c||cc||c|}
\hline$U \backslash A$ & $\mathrm{a}$ & $\mathrm{b}$ & $\mathrm{e}$ \\
\hline $\mathrm{u} 3$ & $a_{1}$ & $b_{2}$ & $e_{2}$ \\
$\mathrm{u} 4$ & $a_{1}$ & $b_{2}$ & $e_{0}$ \\
$\mathrm{u} 5$ & $a_{2}$ & $b_{1}$ & $e_{2}$ \\
$\mathrm{u} 6$ & $a_{2}$ & $b_{1}$ & $e_{2}$ \\
$\mathrm{u} 7$ & $a_{2}$ & $b_{1}$ & $e_{1}$ \\
\hline
\end{tabular}

1. Selecting $\{a\}$

\begin{tabular}{|c||cc||c|}
\hline$U \backslash A$ & $\mathrm{~b}$ & $\mathrm{c}$ & $\mathrm{e}$ \\
\hline $\mathrm{u} 3$ & $b_{2}$ & $c_{0}$ & $e_{2}$ \\
$\mathrm{u} 4$ & $b_{2}$ & $c_{2}$ & $e_{0}$ \\
$\mathrm{u} 5$ & $b_{1}$ & $c_{0}$ & $e_{2}$ \\
$\mathrm{u} 6$ & $b_{1}$ & $c_{1}$ & $e_{2}$ \\
$\mathrm{u} 7$ & $b_{1}$ & $c_{2}$ & $e_{1}$ \\
\hline
\end{tabular}

2. Selecting $\{c\}$

\begin{tabular}{|c||cc||c|}
\hline$U \backslash A$ & $\mathrm{~b}$ & $\mathrm{~d}$ & $\mathrm{e}$ \\
\hline $\mathrm{u} 3$ & $b_{2}$ & $d_{0}$ & $e_{2}$ \\
$\mathrm{u} 4$ & $b_{2}$ & $d_{1}$ & $e_{0}$ \\
$\mathrm{u} 5$ & $b_{1}$ & $d_{0}$ & $e_{2}$ \\
$\mathrm{u} 6$ & $b_{1}$ & $d_{0}$ & $e_{2}$ \\
$\mathrm{u} 7$ & $b_{1}$ & $d_{1}$ & $e_{1}$ \\
\hline
\end{tabular}

3. Selecting $\{d\}$ in the following section.

\subsection{Heuristic Algorithm for Feature Selection}

We use the attributes from CORE as an initial attribute subset. Next, we select one by one an attribute from unselected attributes using some strategies, and we add it to the attribute subset, until a reduct approximation is obtained.

\section{Algorithm.}

Let $R$ be a set of selected condition attributes, $P$ - a set of unselected condition attributes, $U$ - a set of all instances, and EXPECT - an accuracy threshold.

In the initial state, we assume $R=\operatorname{CORE}(C), P=$ $C-\operatorname{CORE}(C), k=0$.

Step 1. Remove all consistent instances:

$$
U=U-P O S_{R}(D)
$$

Step 2. If $k \geq E X P E C T$ where

$$
k=\gamma_{R}(D)=\frac{\operatorname{card}\left(P O S_{R}(D)\right)}{\operatorname{card}(U)}
$$

$$
\begin{aligned}
& \text { then } S T O P \\
& \text { else if } \operatorname{POS}_{R}(D)=P O S_{C}(D) \text { return } \\
& \text { "only } k=\frac{\operatorname{card}\left(P O S_{C}(D)\right)}{\operatorname{card}(U)} \text { is available" }
\end{aligned}
$$

$$
\text { and } S T O P \text {. }
$$

\section{Step 3. Calculate}

$$
\begin{aligned}
& v_{p}=\operatorname{card}\left(P O S_{R \cup\{p\}}(D)\right) \\
& m_{\mathrm{p}}=\max _{-} \operatorname{size}\left(P O S_{R \cup\{p\}}(D)\right) /(R \cup\{p\} \cup D) \\
& \text { for any } p \in P .
\end{aligned}
$$

Step 4. Choose the best attribute $p$, i.e., with the largest $v_{p} \times m_{p}$, and let

$$
R=R \cup\{p\}, P=P-\{p\} ;
$$

Step 5. Go back to Step 2.

Illustrative Example. We select an attribute subset using the above algorithm for a sample database shown in Table 1.

In Table $1, a, b, c$, and $d$ are condition attributes, $e$ is the decision attribute, and $U=\{u 1, u 2, u 3, u 4$, $u 5, u 6, u 7\}, b$ is the unique indispensable attribute (deleting $b$ will cause inconsistency: $\left\{a_{1} c_{2} d_{1}\right\} \rightarrow e_{1}$ and $\left\{a_{1} c_{2} d_{1}\right\} \rightarrow e_{0}$ ).

From the following families of equivalence classes $U /\{b\}=\{\{u 1, u 2\},\{u 5, u 6, u 7\},\{u 3, u 4\}\}$ and $U /\{e\}=\{\{u 4\},\{u 1, u 2, u 7\},\{u 3, u 5, u 6\}\}$, we obtain $\{b\}$-positive region of $\{e\}: \operatorname{POS}_{\{b\}}(\{e\})=$ $\{u 1, u 2\}$. Hence, in the initial state we have $R=\{\mathrm{b}\}$, $P=\{\mathrm{a}, \mathrm{c}, \mathrm{d}\}$, and $U=\{u 3, u 4, u 5, u 6, u 7\}$. The initial state is shown in Table 2.

Assuming $E X P E C T=1$, the termination condition 
will be $k \geq 1$. Since $k=2 / 7<1, R$ is not a reduct, and we must continue to select condition attributes. The next candidates are $a, c$ or $d$. Table 3 gives the results of adding $\{a\},\{c\}$, and $\{d\}$ to $R$, respectively.

From Table 3 we obtain the following families of equivalence classes:

$$
\begin{aligned}
& U /\{e\}=\{\{u 3, u 5, u 6\},\{u 4\},\{u 7\}\}, \\
& U /\{a, b\}=\{\{u 3, u 4\},\{u 5, u 6, u 7\}\}, \\
& U /\{b, c\}=\{\{u 3\},\{u 4\},\{u 5\},\{u 6\},\{u 7\}\}, \\
& \mathrm{U} /\{\mathrm{b}, \mathrm{d}\}=\{\{\mathrm{u} 3\},\{u 4\},\{u 5, u 6\},\{u 7\} .
\end{aligned}
$$

We have also

$$
\begin{aligned}
& \operatorname{POS}_{\{a, b\}}(\{e\})=\emptyset, \\
& P O S_{\{b, c\}}(\{e\})=P O S_{\{b, d\}}(\{e\}) \\
& \quad=\{u 3, u 4, u 5, u 6, u 7\}, \\
& \text { max_size }\left(P O S_{\{b, c\}}(\{e\}) /\{b, c, e\}\right)=1, \\
& \max \text { size }_{-}\left(\operatorname{POS}_{\{b, d\}}(\{e\}) /\{b, d, e\}\right) \\
& \quad=\operatorname{card}(\{u 5, u 6\})=2,
\end{aligned}
$$

One can see that selecting the attribute $a$ we cannot reduce the number of contradictory instances, but if either $c$ or $d$ are chosen then all instances become consistent. Since the maximal set is in $U /\{b, d, e\}$, according to our selection strategies, $d$ should be selected first.

After adding $d$ to $R$, all instances are consistent and must be removed from $U$. Hence $U$ becomes empty, $k=1$, and the process is finished. Thus, the selected attribute subset is $\{\mathrm{b}, \mathrm{d}\}$.

\section{Rough Sets and Boolean Reasoning (RSBR)}

RSBR is a system for discretization of real valued attributes [46]. Discretization of real valued attributes is an important pre-processing step in our rule discovery process. The development of RSBR is based on the following observations: (i) real-life data sets often contain mixed types of data such as real valued, symbolic data, etc.; (ii) real value attributes should be discretized in preprocessing; (iii) the choice of discretization method depends on analyzed data.

The core module in our rule discovery process is GDT-RS. In GDT-RS, the probabilistic distribution between possible instances and possible generaliza-
Table 4: An example of discretization

\begin{tabular}{|c||c|c||c|c|c||c|c||c|}
\hline $\mathrm{U}$ & $\mathrm{a}$ & $\mathrm{b}$ & $\mathrm{d}$ \\
\hline $\mathrm{x} 1$ & 0.8 & 2 & 1 \\
$\mathrm{x} 2$ & 1 & 0.5 & 0 \\
$\mathrm{x} 3$ & 1.3 & 3 & 0 \\
$\mathrm{x} 4$ & 1.4 & 1 & 1 \\
$\mathrm{x} 5$ & 1.4 & 2 & 0 \\
$\mathrm{x} 6$ & 1.6 & 3 & 1 \\
$\mathrm{x} 7$ & 1.3 & 1 & 1 \\
\hline
\end{tabular}

tions depends on the number of values of attributes. Rules induced without discretization are of low quality because usually they will not recognize new objects.

\subsection{Discretization Based on RSBR}

In order to solve the discretization issues, we have developed a discretization system called RSBR that is based on a hybridization of rough sets and Boolean reasoning proposed in [20,21].

A great effort has been made (see e.g. $[5,7,8$, 22]) to find effective methods for discretization of real valued attributes. We may obtain different results by using different discretization methods. The results of discretization affects directly the quality of the discovered rules. Some of discretization methods totally ignore the effect of the discretized attribute values on the performance of the induction algorithm. RSBR combines discretization of real valued attributes and classification together. In the process of the discretization of real valued attributes we should also take into account the effect of the discretization on the performance of our induction system GDT-RS.

Roughly speaking, the basic concepts of the discretization based on RSBR can be summarized as follows: (i) discretization of a decision table, where $V_{c}=\left[v_{c}, w_{c}\right)$ is an interval of real values taken by attribute $c$, is a searching process for a partition $P_{c}$ of $V_{c}$ for any $c \in C$ satisfying some optimization criteria (like minimal partition) preserving some discernibility constraints [20,21]; (ii) any partition of $\mathrm{V}_{c}$ is defined by a sequence of the so-called cuts $v_{1}<v_{2}<\cdots<v_{k}$ from $V_{c}$; (iii) any family of partitions $\left\{P_{c}\right\}_{c \in C}$ can be identified with a set of cuts.

Table 4 shows an example of discretization. The discretization process returns a partition of the 
Table 5: An example of the $T^{p}$

\begin{tabular}{|c||c|c|c|c|c|c|c|}
\hline$U^{*}$ & $p_{1}^{a}$ & $p_{2}^{a}$ & $p_{3}^{a}$ & $p_{4}^{a}$ & $p_{1}^{b}$ & $p_{2}^{b}$ & $p_{3}^{b}$ \\
\hline (x1, x2) & 1 & 0 & 0 & 0 & 1 & 1 & 0 \\
(x1, x3) & 1 & 1 & 0 & 0 & 0 & 0 & 1 \\
(x1, x5) & 1 & 1 & 1 & 0 & 0 & 0 & 0 \\
(x4, x2) & 0 & 1 & 1 & 0 & 1 & 0 & 0 \\
(x4, x3) & 0 & 0 & 1 & 0 & 0 & 1 & 1 \\
(x4, x5) & 0 & 0 & 0 & 0 & 0 & 1 & 0 \\
(x6, x2) & 0 & 1 & 1 & 1 & 1 & 1 & 1 \\
(x6, x3) & 0 & 0 & 1 & 1 & 0 & 0 & 0 \\
(x6, x5) & 0 & 0 & 0 & 1 & 0 & 0 & 1 \\
(x7, x2) & 0 & 1 & 0 & 0 & 1 & 0 & 0 \\
(x7, x3) & 0 & 0 & 0 & 0 & 0 & 1 & 1 \\
(x7, x5) & 0 & 0 & 1 & 0 & 0 & 1 & 0 \\
\hline
\end{tabular}

value sets of condition attributes into intervals:

$$
P=\{(a, 0.9),(a, 1.5),(b, 0.75),(b, 1.5)\} .
$$

\subsection{An Algorithm}

The main steps of the algorithm can be described as follows:

Step 1. Define a set of Boolean variables $B V(U)$. For the example shown in Table 4 we have $B V$ $(U)=\left\{p_{1}^{a}, p_{2}^{a}, p_{3}^{a}, p_{4}^{a}, p_{1}^{b}, p_{2}^{b}, p_{3}^{b}\right\}$, where

$p_{1}^{a}$ corresponds to the interval $[0.8,1)$ of $a$; $p_{2}^{a}$ corresponds to the interval $[1,1.3)$ of $a$; $p_{3}^{a}$ corresponds to the interval $[1.3,1.4)$ of $a$; $p_{4}^{a}$ corresponds to the interval $[1.4,1.6)$ of $a$; $p_{1}^{b}$ corresponds to the interval $[0.5,1)$ of $b$; $p_{2}^{b}$ corresponds to the interval $[1,2)$ of $b$; $p_{5}^{b}$ corresponds to the interval $[2,3)$ of $b$.

Step 2. Create a new decision table $T^{p}$ by using the set of Boolean variables defined in Setp 1. Here $T^{p}$ is called $P$-discretization of $T, T^{p}=$ $\left(U, \cup\{d\}, A^{p}, d\right), p_{h}^{c}$ is a propositional variable corresponding to the interval $\left[v_{k}^{c}, v_{k+1}^{c}\right)$ for any $k \in\left\{1, \ldots, n_{c}-1\right\}$ and $c \in C$.

Table 5 shows an example of the $T^{p}$. We assume e.g., $\left(p_{1}^{a}\left(x_{1}, x_{2}\right)=1\right.$ because any cut in the interval $[0.8,1)$ corresponding to $p_{1}^{a}$ discerns $x_{1}, x_{2}$.

Step 3. Find the minimal subset of $P$ that discerns all objects in different decision classes by using the discernibility formula

$$
\Phi^{\mathrm{U}}=\wedge\left\{\psi(i, j): d\left(x_{i}\right) \neq d\left(x_{j}\right)\right\}
$$

where $\psi(i, j)$, for example, $\psi(i, j)=p_{1}^{a} \vee p_{1}^{b} \vee p_{2}^{b}$ means that in order to discern object $x_{1}$ and $x_{2}$, at least one of the following cuts must be selected: (i) a cut between $a(0.8)$ and $a(1)$; (ii) a cut between $b(0.5)$ and $b(1)$; (iii) a cut between $b(1)$ and $b(2)$.

We obtain from Table 5 the discernibility formula

$$
\begin{aligned}
\Phi^{U} & =\left(p_{1}^{a} \vee p_{1}^{b} \vee p_{2}^{b}\right) \wedge\left(p_{1}^{a} \vee p_{2}^{a} \vee p_{3}^{b}\right) \\
& \wedge\left(p_{1}^{a} \vee p_{2}^{a} \vee p_{3}^{a}\right) \\
& \wedge\left(p_{2}^{a} \vee p_{3}^{a} \vee p_{1}^{b}\right) \wedge\left(p_{2}^{a} \vee p_{2}^{b} \vee p_{3}^{b}\right) \\
& \wedge\left(p_{2}^{a} \vee p_{3}^{a} \vee p_{4}^{a} \vee p_{1}^{b} \vee p_{2}^{b} \vee p_{3}^{b}\right) \\
& \wedge\left(p_{3}^{a} \vee p_{4}^{a}\right) \wedge\left(p_{4}^{a} \vee p_{3}^{b}\right) \wedge\left(p_{2}^{a} \vee p_{1}^{b}\right) \\
& \wedge\left(p_{2}^{b} \vee p_{3}^{b}\right) \wedge\left(p_{3}^{a} \vee p_{2}^{b}\right) \wedge p_{2}^{b}
\end{aligned}
$$

Finally, we obtain four prime implicants denoted by the discernibility formula in DNF form,

$$
\begin{aligned}
\Phi^{\mathrm{U}} & =\left(p_{2}^{a} \wedge p_{4}^{a} \wedge p_{2}^{b}\right) \vee\left(p_{2}^{a} \wedge p_{3}^{a} \wedge p_{2}^{b} \wedge p_{3}^{b}\right) \\
& \vee\left(p_{3}^{a} \wedge p_{1}^{b} \wedge p_{2}^{b} \wedge p_{3}^{b}\right) \vee\left(p_{1}^{a} \wedge p_{4}^{a} \wedge p_{1}^{b} \wedge p_{2}^{b}\right)
\end{aligned}
$$

Furthermore, we select $\left\{p_{2}^{a}, p_{4}^{a}, p_{2}^{b}\right\}$, i.e., $P=$ $\{(a, 1.2),(a, 1.5),(b, 1.5)\}$, as the optimal result, because it is the minimal subset of $P$ preserving discernibility.

\section{GDT-RS in Inductive Logic Programming (GDTRS-ILP)}

GDT-RS stated in Section 2 is a system belonging to attribute-value learning, which is a main stream in inductive learning and data mining communities up to date. Another type of inductive learning is relation learning or called Inductive Logic Programming (ILP) $[13,18]$.

ILP is a relatively new method in machine learning. ILP is concerned with learning from examples within the framework of predicate logic. ILP is relevant to data mining, and compared with the attribute-value learning methods, it possesses the following advantages:

- ILP can learn knowledge which is more expressive than that by the attribute-value learning methods, because the former is in predicate logic while the latter is usually in propositional logic. 
- ILP can utilize background knowledge more naturally and effectively, because in ILP the examples, the background knowledge, as well as the learned knowledge are all expressed within the same logic framework.

However, when applying ILP to large real-world applications, we can identify some weak points compared with the attribute-value learning methods, such as:

- It is more difficult to handle numbers (especially continuous values) prevailing in realworld databases, because predicate logic lacks effective means for this.

- The theory, techniques and experiences are much less mature for ILP to deal with imperfect data (uncertainty, incompleteness, vagueness, impreciseness, etc. in examples, background knowledge as well as the learned rules) than in the traditional attribute-value learning methods (see [13, 33], for instance).

The discretization of continuous valued attributes, which is a kind of Granular Computing, as a pre-processing step, is a solution for the first problem mentioned in the above [22]. Another way is to use Constraint Inductive Logic Programming (CILP), an integration of ILP and CLP (Constraint Logic Programming) [15].

For the second problem, a solution is to combine GDT (also GDT-RS) with ILP, that is, GDT-ILP and GDTRS-ILP to deal with some kinds of imperfect data which occur in large real-world applications [16].

\subsection{The Normal Problem Setting for ILP}

We follow the notations of [19]. Especially, supposing $C$ is a set of clauses $\left\{c_{1}, c_{2}, \ldots\right\}$, we use $\bar{C}$ to denote the set $\left\{\neg c_{1}, \neg c_{2}, \ldots\right\}$. The normal problem setting for ILP can be stated as follows:

Given the positive examples $E^{+}$and the negative examples $E^{-}$(both are sets of clauses) and the background knowledge $B$ (a finite set of clauses),
ILP is to find a theory $H$ (a finite set of clauses) which is correct with respect to $E^{+}$and $E^{-}$. That demands:

1. $\forall{ }_{e \in E^{+}} H \cup B \mid=e$ (completeness wrt. $E^{+}$);

2. $H \cup B \cup \overline{E^{-}}$is satisfiable

(consistency wrt. $E^{-}$).

The above ILP problem setting is somewhat too general. In most of the ILP literature, the following simplifications are assumed:

- Single predicate learning. The concept to be learned is represented by a single predicate $p$ (called the Target predicate). Examples are instances of the target predicate $p$ and the induced theory is the defining clauses of $p$. Only the background knowledge $B$ may contain definitions of other predicates which can be used in the defining clauses of the target predicate.

- Restricted within definite clauses. All clauses contained in $B$ and $H$ are definite clauses, and the examples are ground atoms of the target predicate. We can prove:

supposing that $\Sigma$ is a set of definite clauses, $E^{-}$ is a set of ground atoms, then $\Sigma$ is consistent with respect to $E^{-}$if and only if $\forall_{e \in \mathrm{E}^{-}} \Sigma \mid \neq e$.

So in this case the second condition of correctness (consistency: $H \cup B \cup \overline{E^{-}}$is satisfiable) can be replaced by a simpler form $\forall_{e \in \mathrm{E}^{-}} H \cup B \mid \neq e$. Clearly, this simpler form is more operational (easier to test) than the general one.

To make the ILP problem meaningful, we assume the following prior conditions:

1. $B$ is not complete with respect to $E^{+}$. (Otherwise there will be no learning task at all, because the background knowledge itself is the solution).

2. $B \cup E^{+}$is consistent with respect to $E^{-}$(Otherwise there will be no solution to the learning task). 
Note that almost all practical ILP systems impose some restrictions on the hypothesis space and/or on the search strategies, in order to solve real-world problems with reasonable computing resource (time and/or space). These restrictions are called declarative bias. Bias setting is a key factor for an ILP system to be successful in practice. We can cite the famous statement: "No Bias, no prediction" [17]. Without bias or with too weak bias an ILP system may show poor performance or produce too many solutions that are not interesting to the user. On the other hand, if the bias is too strong, we may miss some useful solutions or have no solution at all.

In the above normal problem setting of ILP, everything is assumed correct and perfect. But in large, real-world empirical learning, data are not always perfect. In contrary, uncertainty, incompleteness, vagueness, impreciseness, etc. are frequently observed in the input to ILP - the training examples and/or background knowledge. Imperfect input, in addition to improper bias setting, will induce imperfect hypotheses. Thus ILP has to deal with imperfect data. In this aspect, the theory, techniques, measurement and experiences are much less mature in ILP than in the traditional attributevalue learning methods (compare with [33], for example).

\subsection{GDTRS-ILP}

At the current stage, we apply GDT and rough set theory to ILP to deal with some kinds of imperfect data occurring in large real-world applications [16]. Namely, we concentrate on incomplete background knowledge (where essential predicates/ clauses are missing), indiscernible data (where some examples belong to both sets of positive and negative training examples), missing classification (where some examples are unclassified) and too strong declarative bias (hence the failure in searching for solutions). Although imperfect data handling is a too vast task, we observe that many problems concerning imperfect input or too strong bias in ILP have a common feature. In these situations, while it is impossible to differentiate distinct objects, we may consider granules - sets of objects drawn together by similarity, indistinguishability, or fun- ctionality. The emerging theory of Granular Computing $(G r C)$ (see $[35,36,37]$ ) grasps the essential concept - granules, and makes use of them in general problem solving. The main idea is that, when we use granules instead of individual objects, we are actually relaxing the strict requirements in the standard normal problem setting for ILP. Based on such idea, the GDTRS-ILP system is under development.

\section{Concluding Remarks}

We have presented several rough sets based hybrid systems that can be used in a multi-phase KDD process for discovering classification rules in databases. Some of databases such as optician, slope-collapse, meningitis, postoperative patient, mushroom, earth quake, bacterial examination, cancer have been tested for the rough sets based systems. The results have been evaluated by several different domain experts $[42,44,45,46]$. The rule discovery process described in this paper and the experimental results shows the usefulness of rough set theory in knowledge discovery and data mining.

The KDD process based on rough set theory can be further extended by including granular computing, decomposition of large databases, mining in ordered information tables, and rule discovery in distributed environment $[10,23,28,29,30,34]$. Our paper is realizing the first step toward a rough sets based multi-strategy and multi-agents discovery system.

\section{Acknowledgements}

The authors would like to thank Prof. S. Tsumoto, Prof. H. Nakamura, Mr. K. Kuramoto, and Mr. H. Sakakibara for providing the medical databases, a slope-collapse database, background knowledge, and evaluating the experimental results. The authors also would like to thank Prof. K. Inoue, Prof. C. N. Liu, Prof. A. Skowron, Prof. Y. Y. Yao, Prof. S. Ohsuga, Prof. M. Inuiguchi, Dr. J. Z. Dong for their valuable comments and helps.

\section{References}

[1] Agrawal, R., Mannila, H., Srikant, R., Toivonen, H., Verkano, A., "Fast Discovery of Association Rules", in: Fayyad U. M., Piatetsky-Shapiro G., 
Smyth P., Uthurusamy R. (eds.) Advances in Knowledge Discovery and Data Mining, The MIT Press (1996) 307-328.

[2] Banerjee, M., Mitra, S., and Pal, S. K. "Rough Fuzzy MLP: Knowledge Encoding and Classification", IEEE Tran. Neural Networks, Vol.9, No.6 (1998) 1203-1216.

[3] Bazan, J. G. "A Comparison of Dynamic and Non -dynamic Rough Set Methods for Extracting Laws from Decision System" in: Polkowski, L., Skowron, A. (Eds.), Rough Sets in Knowledge Discovery 1: Methodology and Applications, Physica-Verlag (1998) 321-365.

[4] Bazan, J. G. and Szczuka, M. "RSES and RSESlib - A Colllection of Tools for Rough Set Computations", Proc. 2nd International Conference on Rough Sets and Current Trends in Computing (RSCTC-2000) (2000) 74-81.

[5] Chmielewski, M. R. and Grzymała-Busse, J. W. "Global Discretization of Attributes as Preprocessing for Machine Learning”, Proc. Thrid Inter. Workshop on Rough Sets and Soft Computing (1994) 294-301.

[6] Dong, J. Z., Zhong, N., and Ohsuga, S. "Probabilistic Rough Induction: The GDT-RS Methodology and Algorithms", in: Z. W. Ras and A. Skowron (eds.), Foundations of Intelligent Systems, LNAI 1609, Springer (1999) 621-629.

[7] Dougherty, J, Kohavi, R., and Sahami, M. "Supervised and Unsupervised Discretization of Real Features", Proc. 12th Inter. Conf. on Machine Learning (1995) 194-202.

[8] Fayyad, U. M. and Irani, K. B. "On the Handling of Real-Valued Attributes in Decison Tree Generation", Machine Learning, Vol. 8 (1992) 87-102.

[9] Fayyad, U. M., Piatetsky-Shapiro, G, and Smyth, P. "From Data Mining to Knowledge Discovery: an Overview", in: U. Fayyad, G. Piatetsky-Shapiro (eds.) Advances in Knowledge Discovery and Data Mining, MIT Press (1996) 1-36.

[10] Greco S., Matarazzo B., and Slowinski R., "Rough Approximation of A Preference Relation by Dominance Relations", European Journal of Operational Research, Vol. 117 (1999) 63-83.

[11] Grzymała-Busse, J. W. "Applications of Rule Induction System LERS”, in: L. Polkowski, A. Skowron (Eds.) Rough Sets in Knowledge Discovery 1: Methodology and Applications, PhysicaVerlag (1998) 366-375.

[12] Komorowski, J., Pawlak, Z., Polkowski, L. and Skowron, A. Rough Sets: A Tutorial, S. K. Pal and A. Skowron (eds.) Rough Fuzzy Hybridization: A New Trend in Decision Making, Springer (1999) 3 $-98$.

[13] Lavrac, N., Dzeroski, S., and Bratko, I. "Handling Imperfect Data in Inductive Logic Programming", L. de Raedt (ed.) Advances in Inductive Logic Programming, IOS Press (1996) 48-64.

[14] Lin, T. Y. and Cercone, N. (ed.) Rough Sets and
Data Mining: Analysis of Imprecise Data, Kluwer (1997).

[15] Liu, C., Zhong, N., and Ohsuga, S. (1997) "Constraint ILP and Its Application to KDD", Proc. of IJCAI-97 Workshop on Frontiers of ILP, 103104.

[16] Liu, C. and Zhong, N. "Rough Problem Settings for ILP Dealing with Imperfect Data", Computational Intelligence, An International Journal, Vol. 17, No. 3, Blackwell Publishers (2001) 446-459.

[17] Mitchell, T., M. Machine Learning, Mc Graw-Hill (1997).

[18] Muggleton, S. "Inductive Logic Programming", New Generation Computing, Vol. 8, No. 4 (1991) 295-317.

[19] Nienhuys-Cheng, S-H. and Wolf, R. de. Foundations of Inductive Logic Programming, LNAI 1228, Springer (1997)

[20] Nguyen, H. Son, Skowron, A. "Quantization of Real Value Attributes", P. P. Wang (ed.) Proc International Workshop on Rough Sets and Soft Computing at Second Joint Conference on Information Sciences (JCIS'95) (1995) 34-37.

[21] Nguyen, H. Son, Skowron, A. "Boolean Reasoning for Feature Extraction Problems", Z. W. Ras, A. Skowron (eds.), Foundations of Intelligent Systems, LNAI 1325, Springer (1997) 117-126.

[22] Nguyen H. Son and Nguyen S. Hoa "Discretization Methods in Data Mining", L. Polkowski, A. Skowron (eds.) Rough Sets in Knowledge Discovery, Physica-Verlag (1998) 451-482.

[23] Nguyen S. H., Nguyen, H. S., Skowron, A. "Decomposition of Task Specification Problems", Z. W. Ras and A. Skowron (eds.) Foundations of Intelligent Systems, LNAI 1609, Springer (1999) 310-318.

[24] Ohsuga, S. "Symbol Processing by Non-Symbol Processor", Proc. 4th Pacific Rim Inter. Conf. on Artificial Intelligence (PRICAI'96) (1996) 193205.

[25] Pal, S. K. and Skowron, A. (Eds.) Rough Fuzzy Hybridization, Springer (1999).

[26] Pawlak, Z. "Rough Sets", International Journal of Computer and Information Sciences, Vol. 11 (1982) 341-356.

[27] Pawlak, Z. Rough Sets, Theoretical Aspects of Reasoning about Data, Kluwer (1991).

[28] Polkowski, L., Skowron, A. "Rough Mereology: A New Paradigm for Approximate Reasoning", International J. Approximate Reasoning, Vol. 15 (4) (1996) 333-365.

[29] Polkowski, L., Skowron, A., "Towards adaptive calculus of granules", L. A. Zadeh and J. Kacprzyk (eds.) Computing with Words in Information/ Intelligent Systems 1: Foundations, Physica-Verlag (1999) 201-228.

[30] Sai, Y., Yao, Y. Y., and Zhong, N. "Data Analysis and Mining in Ordered Information Tables", Proc. 
2001 IEEE International Conference on Data Mining (IEEE ICDM'O1), IEEE Computer Society Press (2001)

[31] Skowron, A. and Rauszer, C. "The Discernibility Matrixes and Functions in Information Systems", R. Slowinski (ed.) Intelligent Decision Support, Kluwer (1992) 331-362.

[32] Tsumoto, S. "Modelling Medical Diagnostic Rules Based on Rough Sets", Rough Sets and Current Trends in Computing, LNAI 1424, Springer (1998) 475-482.

[33] Yao, Y. Y. and Zhong, N. "An Analysis of Quantitative Measures Associated with Rules", Proc. PAKDD'99, LNAI 1574, Springer (1999) 479-488.

[34] Yao, Y. Y. and Zhong, N. "Potential Applications of Granular Computing in Knowledge Discovery and Data Mining", Proc. 5th Inter. Conf. on Information Systems Analysis and Synthesis (IASA' 99) (1999) 573-580.

[35] Yao, Y. Y. "Granular Computing: Basic Issues and Possible Solutions", Proc. JCIS 2000, invited session on Granular Computing and Data Mining, Vol. 1 (2000) 186-189.

[36] Zadeh, L. A. "Fuzzy Sets and Information Granularity”, Gupta, N., Ragade, R. and Yager, R. (Eds.) Advances in Fuzzy Set Theory and Applications, North-Holland (1979) 3-18.

[37] Zadeh, L. A. "Toward a Theory of Fuzzy Information Granulation and Its Centrality in Human Reasoning and Fuzzy Logic", Fuzzy Sets and Systems, Elsevier, Vol. 90 (1997) 111-127.

[38] Zhong, N. and Ohsuga, S. "Toward A MultiStrategy and Cooperative Discovery System", Proc. First Int. Conf. on Knowledge Discovery and Data Mining (KDD-95), AAAI Press (1995) 337 -342 .

[39] Zhong, N., Dong, J. Z., and Ohsuga, S. "Data Mining: A Probabilistic Rough Set Approach", L. Polkowski and A. Skowron (eds.) Rough Sets in Knowledge Discovery, Vol. 2, Physica-Verlag (1998) 127-146.

[40] Zhong, N., Skowron, A., and Ohsuga, S. (eds.) New Directions in Rough Sets, Data Mining, and
Granular-Soft Computing, LNAI 1711, Springer (1999).

[41] Zhong, N., Dong, J. Z., and Ohsuga, S. "Using Background Knowledge as a Bias to Control the Rule Discovery Process", Djamel A. Zighed, Jan Komorowski, and J. Zytkow (eds.) Principles of Data Mining and Knowledge Discovery. LNAI 1910, Springer (2000) 691-698.

[42] Zhong, N., Dong, J. Z., and Ohsuga, S. "Meningitis Data Mining by Cooperatively Using GDT-RS and RSBR", Proc. JSAI 2001 International Work shop on KDD Challenge (JKDD'O1) (2001) 55-60.

[43] Zhong, N., Liu, C., and Ohsuga, S. "Dynamically Organizing KDD Process", International Journal of Pattern Recognition and Artificial Intelligence, Vol. 15, No. 3, World Scientific (2001) 451-473.

[44] Zhong, N., Dong, J. Z., and Ohsuga, S. "Rule Discovery by Soft Induction Techniques",Neurocomputing, An International Journal, Vol. 36 (14) Elsevier (2001) 171-204.

[45] Zhong, N., Dong, J. Z., and Ohsuga, S. "Using Rough Sets with Heuristics to Feature Selection”, Journal of Intelligent Information Systems, Vol. 16, No. 3, Kluwer (2001) 199-214.

[46] Zhong, N. and Skowron, A. "A Rough Sets Based Knowledge Discovery Process”, International Journal of Applied Mathematics and Computer Science, Vol. 11, No. 3, Technical University Press, Poland (2001) 101-117.

[47] Zhong, N. Knowledge Discovery and Data Mining, in the Encyclopedia of Microcomputers, Volume 27 (Supplement 6), Marcel Dekker (2001) 235285.

[問い令わせ先]

(2001年 8 月 $31 \mathrm{H}$ 受付)

Ү371-0816

群馬県前橋市上住鳥町460-1

前橋 [科大学大学院工学研究科

システム情報亡学専攻教授

鍾 寧(Ning $Z$ HONG)

TEL\&FAX : 027-265-7366

E-mail : zhong@maebashi-it.ac.jp

\section{Information about Author}

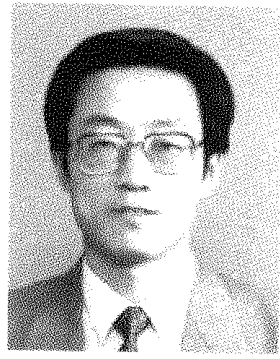

Ning Zhong is currently a professor in Department of Information Engneering, Maebashi Institute of Technology, Japan. He received Ph.D. degree from the University of Tokyo. He has conducted research in the areas of knowledge discovery and data mining, rough sets and granular-soft computing, intelligent agents and databases, web intelligence, knowledge and hybrid systems, with over 100 journal and conference publications and 6 books. He is an editor of Knowledge and Information Systems: an international journal (Springer), a member of the editorial board of Advances in Data Management Book Series (WIT Press). He is a member of the Steering Committee of IEEE International Conference on Data Mining, ACM SIGKDD International Liaisons Board, advisory board of International Rough Set Society. He has also served or is currently serving as guest editors of special issues for several international journals including IEEE Computer. 